\title{
Noise in raw data from magnetic observatories
}

\author{
Sergey Y. Khomutov ${ }^{1}$, Oksana V. Mandrikova ${ }^{1}$, Ekaterina A. Budilova ${ }^{1,2}$, Kusumita Arora ${ }^{3}$, and Lingala Manjula ${ }^{3}$ \\ ${ }^{1}$ Institute of Cosmophysical Research and Radio Wave Propagation FEB RAS, Mirnaya str, 7, \\ Paratunka 684034, Kamchatka, Russia \\ ${ }^{2}$ Kamchatka State Technical University, Klyuchevskaya str, 35, Petropavlovsk-Kamchatsky 683003, Russia \\ ${ }^{3}$ CSIR - National Geophysical Research Institute, Uppal Road, Hyderabad-500007, Telangana, India
}

Correspondence to: Sergey Y. Khomutov (khomutov@ikir.ru)

Received: 28 February 2017 - Discussion started: 10 May 2017

Revised: 3 August 2017 - Accepted: 20 August 2017 - Published: 15 September 2017

\begin{abstract}
In spite of significant progress in the development of new devices for magnetic measurements, mathematical and computational technologies for data processing and means of communication, the quality of magnetic data accessible through the data centres (for example, World Data Centres or INTERMAGNET) still largely depends on the actual conditions in which observation of the Earth's magnetic field is performed at observatories. Processing of raw data of magnetic measurements by observatory staff plays an important role. It includes effective identification of noise and elimination of its influence on final data. In this paper, on the basis of the experience gained during long-term magnetic monitoring carried out at the observatories of IKIR FEB RAS (Russia) and CSIR-NGRI (India), we present a review of methods commonly encountered in actual practice for noise identification and the possibility of reducing noise influence.
\end{abstract}

\section{Introduction}

Magnetic measurements at observatories are an important source of information for studying the processes in the Earth's interior and near-Earth environment that substantially supplements the data obtained from satellites during magnetic surveys or from temporary stations. Currently, about 130 magnetic observatories are integrated into the global observation network INTERMAGNET (www.intermagnet. org), which established the standards for measurements, processing and transmission of the data. INTERMAGNET standards define the requirements for technical parameters of variational and absolute observations, set the requirements for the accuracy of final data (INTERMAGNET Tech. Ref.
Manual, 2012) and specify some noise characteristics of the raw data (Turbitt et al., 2014). However, the requirements for the detection and processing of some types of noise, such as spikes or jumps, are addressed at the level of individual observatories. Final data from INTERMAGNET observatories (quasi-definitive and definitive) undergo multistage control, but their validity and reliability greatly depend on the quality of the raw results of magnetic measurements.

There are a lot of reasons as to why the data quality decreases, for example methodological and hardware (technical) problems, organizational difficulties and noise caused by the environment. Methodological issues are solved in large part by standard requirements according to which measurements are carried out (for example, INTERMAGNET standards in INTERMAGNET Tech. Ref. Manual, 2012) or in guides for organization of magnetic measurements (Jankowski and Sucksdorff, 1996; Nechaev, 2006). Hardware problems are mainly solved by the developers at the level of production (development and production of magnetometers) and partly by a user at an observatory (calibration, comparisons, etc.). As a result of the influence of many external sources, noise manifests as signals in the magnetic field, which are recorded by magnetometers. Considerable parts of papers on noise in magnetic measurements are devoted to hardware noise or noise from uncontrolled sources. The first type is oriented to the developers, and in many cases developers are authors and co-authors (see, for example Denisov et al., 2006; Hegymegi et al., 2016; Khomutov et al., 2016). The second type, in turn, represents almost the whole of scientific research, with the results of an investigation of noise properties, physics of their sources, etc. (see Maule at al., 2009; Neska et al., 2013; Santarelli et al., 2014). At the same 
time, both the first and the second types of paper are not often given the practical recommendations to magnetologists that is, specialists at observatories, who directly make magnetic measurements and process raw data.

Therefore, it seems necessary to make a review of manmade disturbances, which are most frequently encountered in the raw magnetic data and to show examples of possible methodological and software techniques that allow us to eliminate the noise with varying efficiency. As the initial data for the analysis, we considered the results of magnetic measurements, which are carried out at INTERMAGNET observatories: Paratunka, Magadan, Khabarovsk (Russia) and Hyderabad (India). In addition, data from the Cape Schmidt and Choutuppal observatories were used.

The topicality of the information is confirmed by the following:

1. A new generation of magnetologists ensures that all possible problems at the observatories can be solved by modern technologies. At the same time, the lack of experience of real work at magnetic observatories and the absence of full information about actual conditions of measurements can lead to serious negative consequences.

2. Due to the specific nature of the subject (mainly discussed by a narrow circle of specialists, who monitor the magnetic field at the observatories), the results and the conclusions of these discussions remain inaccessible through publications, and in the best case, they appear in the conference proceedings, i.e. are limited in distribution.

3. The amount of data with which scientists have to deal has grown significantly. It is almost impossible to perform a sufficiently correct estimation of the quality of these data, because we deal with the final results of measurements carried out at the observatories, and there is no information indicating the nature of potential problems in this data.

The term "noise" is relative and significantly depends on the specific problems to be solved, used equipment, requirements of accuracy and time resolution, and so on. For example, temporary signals in the magnetic field caused by the sources in the ionosphere-magnetosphere are considered as noise during ground magnetic survey and interpretation of its results. Concerning the magnetic measurements at INTERMAGNET observatories, the signals, which have sources closer than a few tens of kilometres, can be conventionally considered noise (see, for example, Santarelli et al., 2014). Of course, there are some powerful sources of manmade noise such as DC railways, which in the case of appropriate conductivity of the upper layer of the Earth's crust can produce a significant effect in the magnetic data at large distances. On the other hand, some natural phenomena can have smaller spatial sizes than the limit value given above, for example tectonomagnetic and seismomagnetic effects with typical distances to the suspected source of tens of kilometres.

An indication of recognition of the signal as noise can provide the typical duration of this signal. We can be assume that this time is generally not significant, for example, about $1 \mathrm{~h}$ and shorter. At the same time, it is necessary to keep in mind that there are many natural signals with the considered durations. There are also many examples of noise with spectrums similar to natural signals, which can last from days up to months. However, even such random noise often represents a mixture (superposition) of many signals which have much shorter characteristic time of existence (seconds or minutes) and clear structure. For example, railway signals in magnetic measurements have a pulse or rectangular shape at a short distance from the railway (Neska et al., 2013). Therefore, the efficiency of the time criterion for an estimation of the noise is low.

In this work we will classify noise, which is encountered most frequently in actual magnetic measurements at the observatories of IKIR FEB RAS and CSIR-NGR, and illustrate them with some characteristic examples. Naturally, the description and the samples are quite limited, since the variety of noise is extremely large. We will consider only the noise, the man-made nature of which has already been proved or the structure of which allows us to interpret it unambiguously. Magnetic signals with features of noise, but with unknown sources, are not in the scope of this work. The data used in this paper were processed using the tools of a MATLAB mathematical software package (www.mathworks.com) and by application software applied to MATLAB and Octave (http://www.gnu.org/software/octave/) environments used at observatories.

\section{Initial data, description of observatories}

In this paper we apply the data collected during regular magnetic measurements at the observatories of IKIR FEB RAS (Russia) and CSIR-NGRI (India). The observatories are listed in Table 1, their location is shown in Fig. 1. Table 1 shows the name of each observatory, IAGA code, geographical coordinates, institute and status in INTERMAGNET network, and magnetometers.

All listed observatories, with the exception of the Hyderabad observatory (HYB), are located far enough from big cities, but in the vicinity of small settlements. There are no powerful sources of potential noise, such as factories and railways nearby (up to $10-30 \mathrm{~km}$ ). HYB is located within the city and an above-ground subway line has been recently built a few hundreds of metres from the pavilions. Russian observatories CPS, MGD, PET and KHB were built in the 1960s according to the requirements for complex magneticionospheric stations in the USSR, with a wide range of geophysical observations. As a result, there are other observa- 


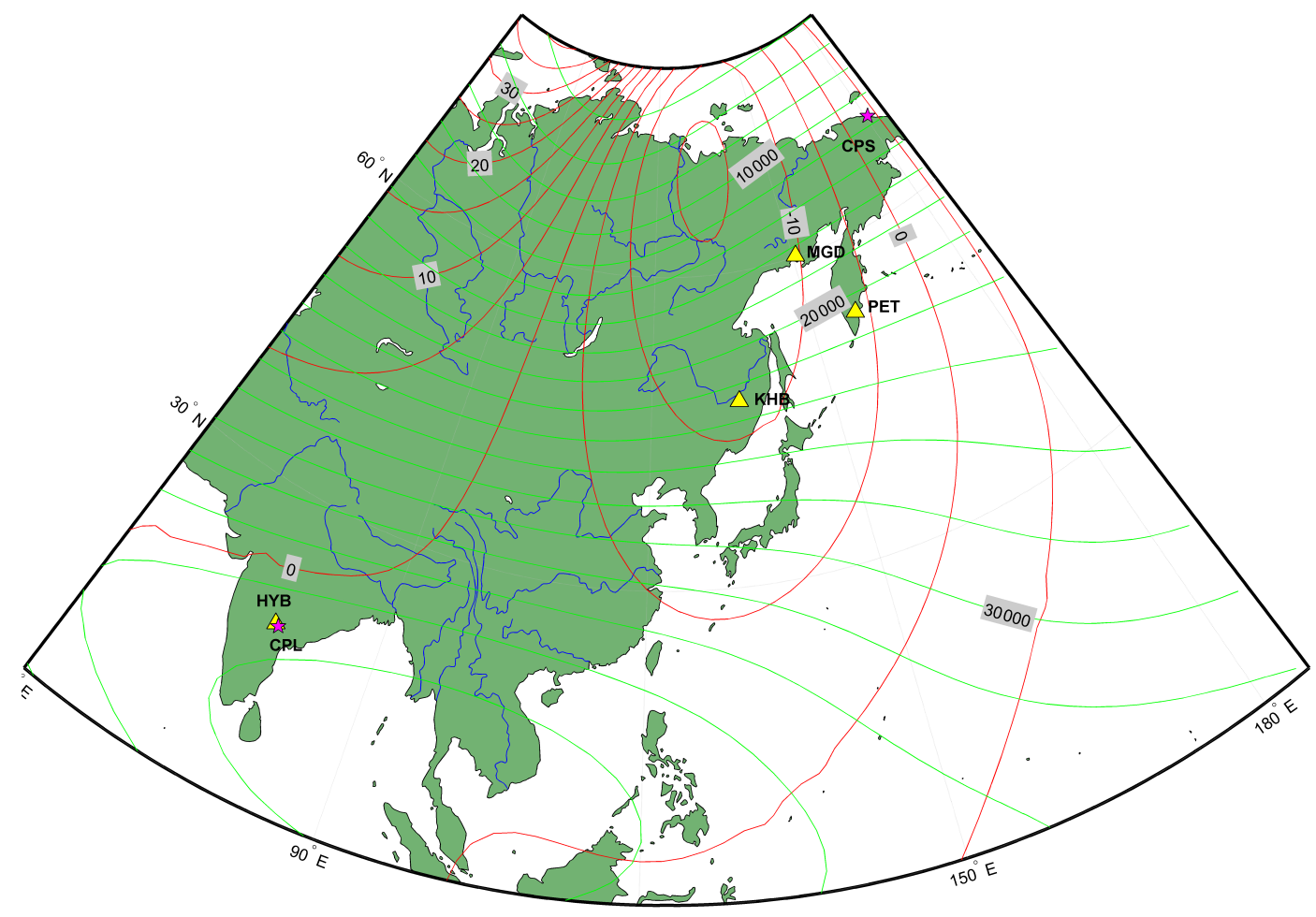

Figure 1. Location of magnetic observatories of IKIR FEB RAS (CPS, KHB, MGD and PET) and CSIR-NGRI (HYB and CPL). Isolines show horizontal component $H$ (green lines) and declination $D$ (red lines) according to IGRF12 model. Yellow triangles are the INTERMAGNET observatories, red stars are non-INTERMAGNET observatories.

Table 1. List of magnetic observatories, the data of which are used in this article. The magnetometers are marked by normal font for vector devices and italic font for scalar ones. IMO is INTERMAGNET magnetic observatory.

\begin{tabular}{llrrll}
\hline Observatory & IAGA & Lat(N) & Lon(E) & Institute & Magnetometers \\
\hline Cape Schmidt & CPS & 68.9 & 180.6 & IKIR & dIdD, Magdas, POS-1 \\
Magadan & MGD & 60.1 & 150.7 & IKIR, IMO & FGE, FRG-601, Magdas, dIdD, GSM-90, POS-1 \\
Paratunka & PET & 53.0 & 158.2 & IKIR, IMO & FGE, FRG-601, Magdas, dIdD, POS-4, GSM-90, POS-1 \\
Khabarovk & KHB & 47.6 & 134.7 & IKIR, IMO & dIdD, Quartz-06, POS-1, GSM-19W \\
Choutuppal & CPL & 17.3 & 78.9 & NGRI & FGE, GEOMAG-02M, GEOMAG-02MO, GSM-90, GSM-19W \\
Hyderabad & HYB & 17.4 & 78.6 & NGRI, IMO & FGE, GEOMAG-02, GSM-90, GSM-19W
\end{tabular}

(1) GSM-90 (http://www.gemsys.ca/scalar-magnetometers/), GSM-19 (http://www.gemsys.ca/rugged-overhauser-magnetometer/) and POS-1

(http://magnetometer.ur.ru/content/view/15/30/lang,en/) are scalar Overhauser magnetometers. (2) dIdD GSM-19FD (http://www.gemsys.ca/vector-magnetometers/) and POS-4 (Sapunov et al., 2016) are vector magnetometers with Overhauser sensor in coil system. (3) MAGDAS (MAGDAS-A Installation Manual, 2005), FRG-601 (3-component fluxgate magnetometer FRG-601G, 2002), FGE

(http://www.space.dtu.dk/english/research/instruments_systems_methods/3-axis_fluxgate_magnetometer_model_fgm-fge) and GEOMAG-02M (Nelapatla et al., 2017) are fluxgate magnetometers. (4) Quartz-06 is magnetometer with Bobrov's quartz sensors (IZMIRAN, Moscow).

tion systems in the immediate vicinity of magnetic pavilions, which are potential sources of interference, for example ionosondes for vertical sounding of the ionosphere. Moreover, due to the limited area, there are some facilities such as garages with heavy machinery, wells and electricity power equipment near the observatories, which may influence the magnetic measurements.

Extreme climate conditions at IKIR observatories are of great importance. For MGD and KHB observatories, sharply continental climate with seasonal changes of temperature from -40 to $+30^{\circ} \mathrm{C}$ is ordinary. The CPS observatory is located in the High Arctic zone with very hard climate conditions. The PET observatory is characterized by an abundance of precipitation and the level of snow in winter is up to $2 \mathrm{~m}$. These conditions require special and expensive means with which to provide the required temperature conditions in magnetic pavilions. Special machinery is necessary to clear snow from the paths to the pavilions, which may also affect the quality of measurements. We should mention such features, as stability of an external power supply is difficult to 

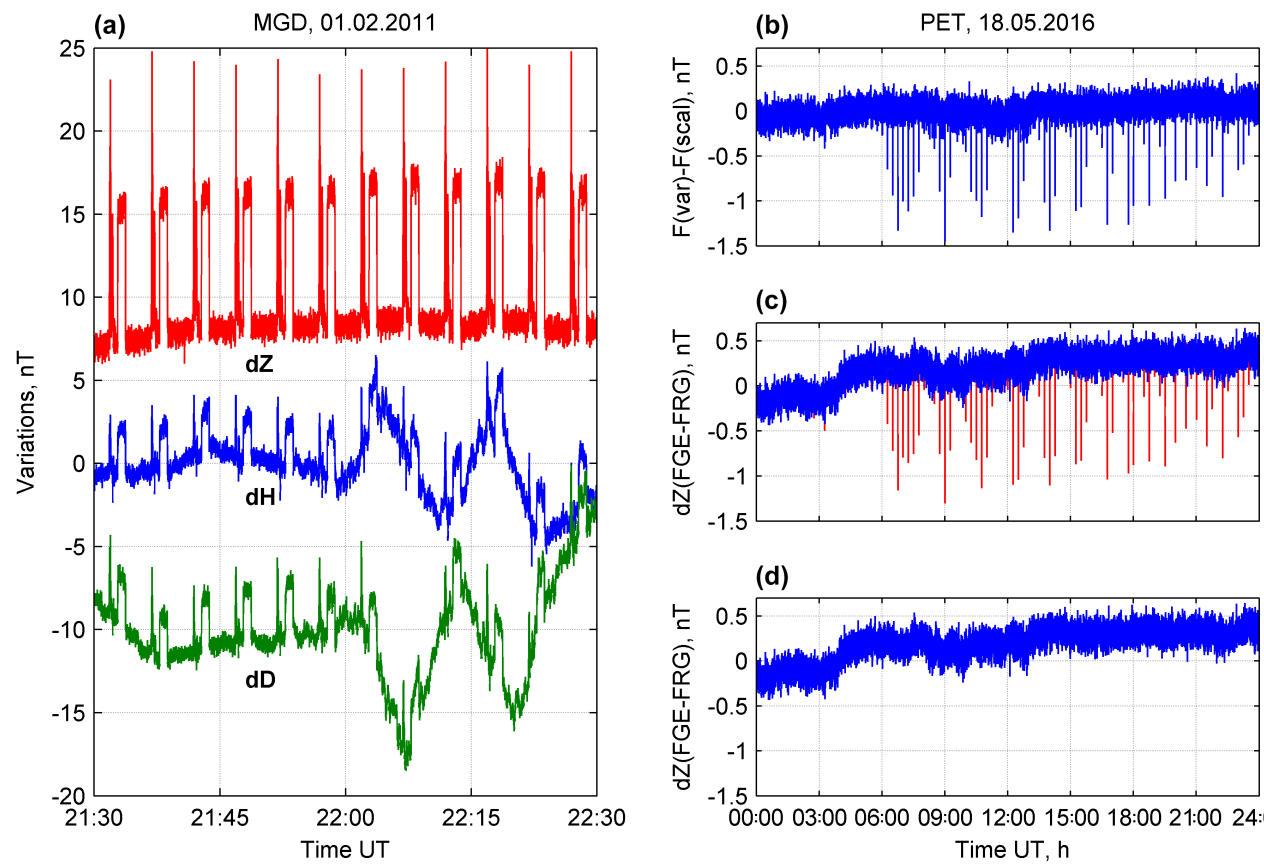

(c)

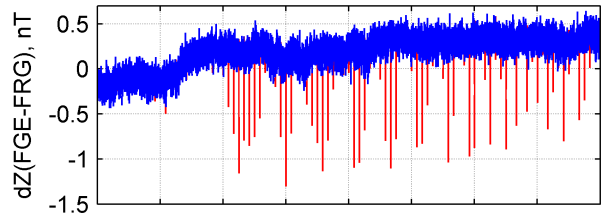

(d)

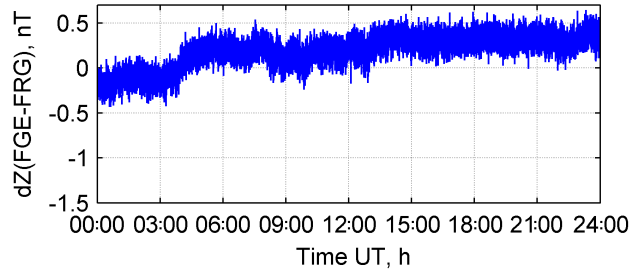

Figure 2. Example of regular noise in magnetic data arising during ionosonde operation. (a) At the Magadan observatory the ionosonde operates with a 5 min periodicity and makes noise signals in all components, recorded by FGE fluxgate magnetometer. (b, c, d) At the Paratunka observatory the ionosonde operates with 15 min periodicity. The effects appear in all records of fluxgate variometer FGE, mainly in the vertical component $Z$. These spikes are visible in the differences between total intensity $F$ (var), calculated from FGE data, and $F$ (scal) calculated from scalar magnetometer GSM-90 (b). Subplot (c) shows the differences between $Z$ records of FGE (with noise from ionosonde) and Japanese fluxgate magnetometer FRG-601, which is not affected by ionosonde activity. Subplot (d) shows the result of clearing the ionosonde effects from FGE records.

provide at the observatories remote from densely populated areas, as well as ground connection quality due to the nature of the ground (at Cape Schmidt and Magadan). For Indian observatories climate conditions are also a significant problem: high temperatures throughout the year and a rainy season with high humidity.

\section{Description and classification of noise in raw data}

\subsection{Regular and random noise}

In order to develop a mechanism to deal with noise effectively, it is necessary to recognize its nature and to classify it as regular and random, frequent and rare, etc. Generally, regular noise is the result of technical problems with measuring equipment or interference from other devices operating in the vicinity of a magnetometer. An indicative example of regular noise is interference in the magnetic data that arises when ionosondes are operating. This is quite a common problem at integrated remote observatories, where it is physically and organizationally impossible to place magnetic and ionospheric measurements at a far enough distance.

Figure 2 (left panel) shows the hourly record from the Magadan observatory obtained by fluxgate magnetometer
FGE with Magdalog datalogger. It contains noise caused by ionosonde operation with regular sessions every $5 \mathrm{~min}$. These spikes have quite large amplitude and duration and produce significant noise in the minute data obtained by averaging using the Gaussian filter (INTERMAGNET standard). A similar picture of interference caused by an ionosonde during vertical sounding every $15 \mathrm{~min}$ is also visible on the daily record obtained by a similar magnetometer at the Paratunka observatory; see Fig. 2 (right panel). Since the noise amplitude does not exceed $2 \mathrm{nT}$, in order to detect it, natural variations of the field were eliminated according to the data from other magnetometers installed in the same pavilion: $Z$ component variation was removed using the data of the fluxgate device FRG-601 (Fig. 2c), $F$ (scal) variation was removed using the data from scalar magnetometer GSM-90 and $F$ (var) was calculated from the FGE variations and the corresponding baseline values (Fig. 2b). The authors have also observed similar interferences in the raw data from Novosibirsk (NVS, fluxgate magnetometer LEMI-008) and Yakutsk (YAK, FGE magnetometer and Magdalog recorder) observatories. The distance to ionosondes did not exceed 200-300 m.

In all cases, the interference was recorded by variation fluxgate magnetometers. At the same time, in the records of the other fluxgate devices located in immediate proximity, interference from the ionosonde was not observed, for exam- 
ple in the results of the fluxgate magnetometer FRG-601, the data of which were used to remove natural geomagnetic variations (illustrated by the difference between the data obtained by FGE and FRG magnetometers shown in Fig. 2c and d). It should be noted that the interference is regular, because it is determined by sounding sessions, the beginning of which is synchronized with UTC. However, small shifts are observed, which are associated with a particular mode of sounding and the range of operating frequencies of the ionosonde.

Regularity and accurate synchronization of such interference with UTC makes it easier to identify them and to make a decision about the removal or correction. Certainly, this applies to relatively short noise signals, usually to spikes. A special software module was developed and integrated into the software package for raw magnetic data processing at the MGD and PET observatories. The module generates a temporary mask, a daily array of 0 and 1 , where 0 corresponds to the measurements that include noise. Parameters of the $0-1$ sequence are set in a special text file that contains the periodicity of sounding and the shifts of the beginning and the end of a removed interval with respect to the beginning of a sounding session for each day. During the processing of the raw data, the mask is applied to the original daily time series and the data marked by " 0 " are filled with special values $\mathrm{NaN}$ (Not-a-Number). An example of this clearing is shown in Fig. 2d.

Interference between closely spaced magnetometers may be a source of regular noise. It is a well known that proton magnetometers can cause interference either by the generation of additional external magnetic fields during polarization of the proton rich liquid or via direct influence of the DC current powering the proton (or Overhauser effect) magnetometers, which is modulated by the periodicity of measurements. For example, the effect up to $0.2 \mathrm{nT}$ is expected at a distance of about $5 \mathrm{~m}$ from the proton magnetometer (Auster et al., 2007). Vector magnetometers using proton sensors in the coil systems (dIdD GSM-19FD, GEM System; POS-4, QMLab), in addition to the effects during polarization, produce significant additional magnetic fields affecting the measurements of devices located in the vicinity. Figure $3 \mathrm{a}$ and b show manifestation of the dIdD magnetometer operation at the Cape Schmidt observatory in the records of $\mathrm{d} H$ and $\mathrm{d} D$ variations from fluxgate magnetometer MAGDAS. Due to the polar specifics (necessity of heating and the absence of additional pavilions) both devices are installed on one pillar at a distance of about $2 \mathrm{~m}$, almost on the same meridian. MAGDAS measurement frequency is $1 \mathrm{~Hz}$. Oscillations in a range up to $2 \mathrm{nT}$ with a period that is a multiple of dIdD measurement periodicity $(2.5 \mathrm{~s})$ are observed. There is also noise in the record of vertical component. The mechanism of influence of dIdD on MAGDAS measuring process is complex: it is associated with timer stability (noise amplitude "floats" over time) and practically it cannot be reliably corrected by software during the post-processing. Therefore, the only ef-
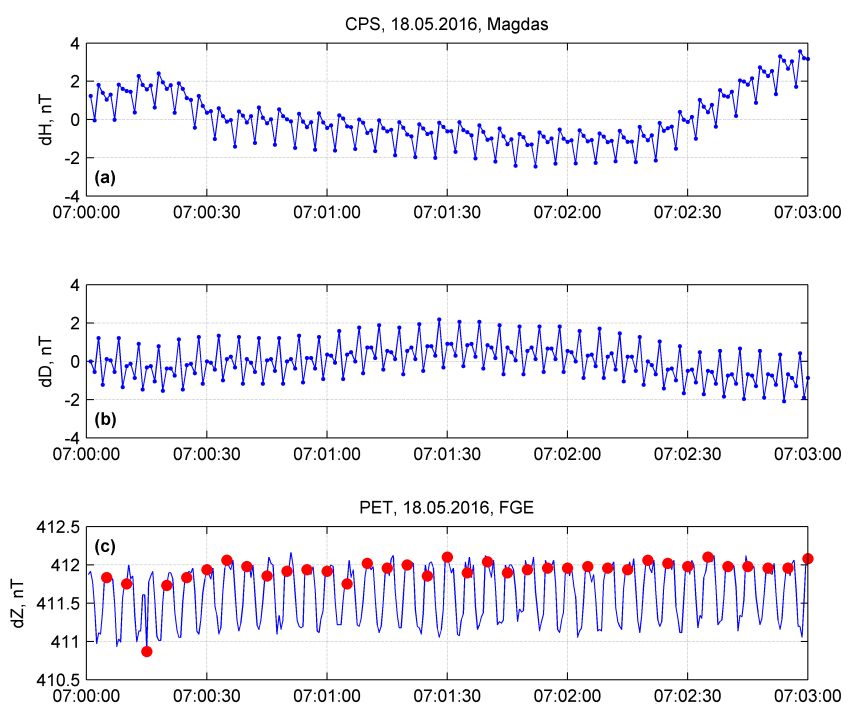

Figure 3. An example of influence of the magnetometers with Overhauser scalar sensors: $(\mathbf{a}, \mathbf{b})$ effect of vector dIdD GSM-19FD in the records of $H$ and $D$ components of MAGDAS fluxgate variometer at the Cape Schmidt observatory, (c) influence of the scalar magnetometer GSM-90 on the vertical component $Z$ of the FGE fluxgate variometer at the Paratunka observatory. Oscillations of $d Z$ have the period of GSM-90 measurements, undisturbed $\mathrm{d} Z$ are found at upper part of oscillations; red dots show undisturbed values, which are used for the next processing step.

fective way to avoid this interference is to distance the magnetometers from each other.

Figure $3 \mathrm{c}$ shows the second example of the proton magnetometer influence on another device at the Paratunka observatory. The fluxgate variation magnetometer FGE signal (measuring frequency is $2 \mathrm{~Hz}$ ) is modulated by the operation of a GSM-90 Overhauser magnetometer with a measuring rate of $5 \mathrm{~s}$. The range of noise in the vertical component $Z$ is up to $1-2 \mathrm{nT}$ (in other components it is less than $0.5 \mathrm{nT}$ ). Since the sensor GSM-90 is located at a distance of about $4 \mathrm{~m}$ from the FGE sensor, the impact through additional magnetic fields during polarization is hardly probable. Presumably, the interaction takes place at the hardware or communication level, because the devices are connected by a single datalogger Magdalog. The same noise is also observed on a similar set of magnetometers at the Magadan observatory. We cannot solve this problem technically. However, the synchronicity of measurements by two instruments (on one datalogger with a single timer) has allowed us to implement a software clearing. From the fluxgate magnetometer data we select only those which occurred during the frequency measurement of proton sensor precession, and fragments during polarization are removed. Unfortunately, due to a little synchronicity instability of measurements by two devices, only a few samples from the $5 \mathrm{~s}$ cycle of FGE can be reliably distinguished (for reliability actually only one sample is chosen, 

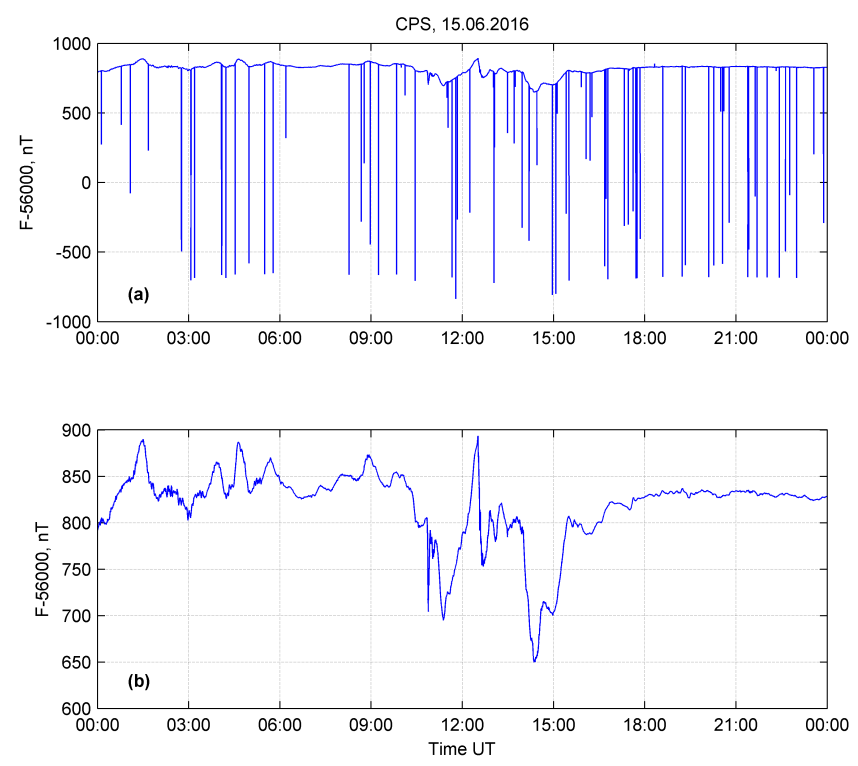

Figure 4. An example of spikes in the results of measurements by Overhauser magnetometer POS-1 at the Cape Schmidt observatory. (a) The total intensity $F$ raw record, (b) signal after noise removal.

which is shown in Fig. $3 \mathrm{c}$ by $\bigcirc$ ), i.e. forced 10 -fold data loss takes place.

In minute values, calculated in accordance with the INTERMAGNET standard, normally distributed noise can (in special cases) be removed by filtering. However, the fact that noise is often asymmetrical creates at least two problems on minute intervals:

a. When averaging the noise with asymmetric signals, the obtained mean values are also biased. For the noise shown in Fig. $3 \mathrm{c}$ with magnitude up to $-1 \mathrm{nT}$, the average estimate will be systematically biased downward by about $0.5 \mathrm{nT}$. This value is quite significant in relation to current requirements for long-term magnetic measurements.

b. If during the absolute observations a reading at the zero position of DIflux magnetometer fluxgate sensor coincides with noise in the variometer, then a difference between absolute and variation measurement arises; i.e. the accuracy of baseline values is decreased, and in the worst cases systematic errors in the final values of the total field vector arise.

\subsection{Noise of different shapes}

\subsubsection{Spikes}

A generalized view of possible shapes of structured noise is presented, for example in Lopez-de-Lacalle (2016, Fig. 1). Spikes are perhaps one of the most common types of signal in magnetic records, and with a sufficient probability are not related to natural processes. Spike is interpreted as relatively short signal with a significant amplitude (duration is less than a few seconds or several measurements, if the magnetometer has a measurement frequency from ones to tenths of $\mathrm{Hz}$ ), with well-defined sharp leading and back edges that are similar in amplitude. If one of the edges is weak or absent, then we can talk about the jump. All these properties of spikes can be used for their detection and removal. We can also note that spikes with a duration of one measurement cycle are most likely to be associated with hardware problems or interferences from nearby sources. The amplitude is also an important characteristic. Spikes with amplitudes of tens of nanotesla or more also have a low probability of being caused by natural sources.

Figure 4a shows an example of a daily record of the field total intensity $F$ recorded at the Cape Schmidt observatory by the Overhauser magnetometer, POS- 1 . The measurement periodicity is $3 \mathrm{~s}$. For the CPS observatory, problems with the stability of the power supply and the quality of grounding are known, to which POS- 1 is sensitive enough. On the record, outliers with the amplitude up to $1500 \mathrm{nT}$ can be seen (over 90 events). The duration of these spikes does not exceed one measurement; i.e. they have sharp edges with almost equal magnitude of leading and back fronts. Therefore, it is not difficult to identify and to locate them. The results from using the simple method of spike detection are shown on Fig. $4 \mathrm{~b}$. It should be noted that in the Overhauser magnetometers POS-1, which are quite widely distributed at magnetic observatories, each record is accompanied by the estimation of measurement quality using a special parameter QMC (quality measurement criterion). The QMC value is related to the quality of the proton precession signal and gives qualitative estimates of measurement conditions such as signal-to-noise ratio, the duration of the precession signal and power supply voltage (POS-1 User manual, 2004; Denisov et al., 2006). Similarly, although a little less informative, estimations of signal quality are also performed for scalar magnetometers GSM (GSM-19 Instruction Manual, 2008, p. 54). For POS-1 these qualification parameters are used in the standard software at IKIR FEB RAS observatories to estimate the quality of measurements within the processing, which increases the efficiency of simple mathematical algorithms.

In many cases the visual control of the derivatives of magnetic field variations is provided by effective tools with which to detect the spikes in recorded raw data. It is clear that noise in the record shown in Fig. 4a does not represent a problem for programme processing, and in the case of smaller quantities they can be processed manually. However, difficulties arise in the case of more irregular shapes of noise, when they cannot be considered narrow isolated spikes. The examples are the noise described above, arising during ionosonde operation (Fig. 2a and b), which is often extended in time and can have a multimodel structure. The efficiency of their processing is provided by strict repeatability. 

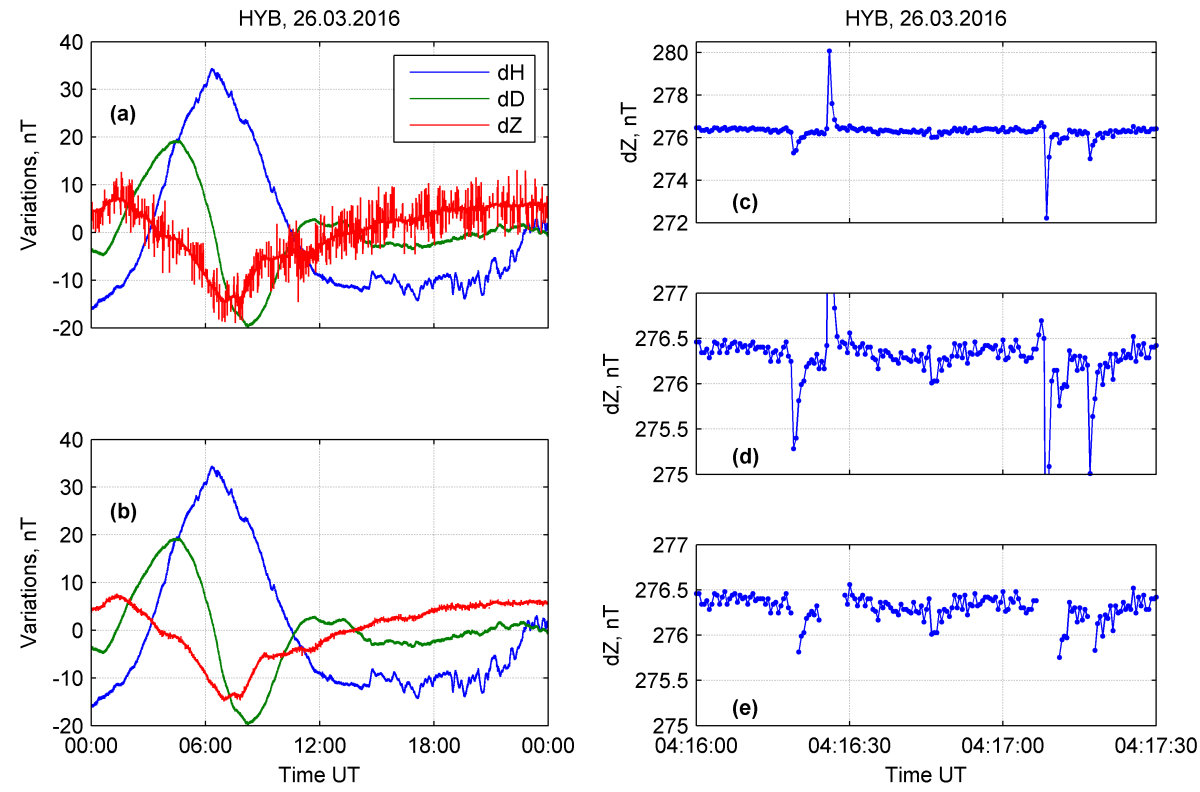

Figure 5. Example of frequent spikes at the Hyderabad observatory: (a) original daily records of variations $\mathrm{d} H, \mathrm{~d} D, \mathrm{~d} Z$; (b) the same of (a), but after noise identification and removal in vertical component; (c) 1.5 min fragment of daily dZ record, showing the detailed structure of the spikes; (d) results of noise clearing using a simple algorithm; (e) result of clearing using an algorithm based on wavelet transform.

Figure 5 shows the daily record of $\mathrm{d} H, \mathrm{~d} D$ and $\mathrm{d} Z$ variations, obtained at the Hyderabad observatory using the fluxgate magnetometer FGE. The measurement frequency is $2 \mathrm{~Hz}$. As can be clearly seen, there is irregular and frequent noise in the vertical component in the form of outliers (spikes) with amplitudes of more than $5 \mathrm{nT}$. The observatory is located on the territory of CSIR-NGRI Institute, within the city. The most probable reason for this noise is the metro line (above-ground) passing at a distance of 200-300 m, south of the observatory. Figure $5 \mathrm{c}$ shows a $1.5 \mathrm{~min}$ fragment for $\mathrm{d} Z$, which shows that spikes have a sufficiently definite and stable structure, a sharp leading edge and exponentially falling back edge. The total spike duration is up to 3-6 samples, i.e. about $1-3 \mathrm{~s}$

The algorithm applied at the HYB observatory to detect and remove the noise caused by metro line is based on its structural stability and works as follows: when a change of $\mathrm{d} Z$ between neighbouring measurements (in absolute value) exceeding a given threshold is found, three subsequent measurements are discarded. It is clear that this algorithm creates risks: (a) natural signals with sharp edges, for example, during magnetic disturbances, may be discarded; (b) noise may be removed not completely if its duration is longer than one; (c) noise can be missed if due to a small shift its leading edge takes two samples. However, to calculate the minute values of the magnetic field variations, that may be enough. Figure $5 \mathrm{~d}$ shows the results of the algorithm application during real processing of raw HYB data (threshold value $\mathrm{d} Z / \mathrm{d} t=1 \mathrm{nT} / 0.5 \mathrm{~s}$ was used).
An algorithm based on continuous wavelet transform showed higher efficiency in the detection of spikes. For the first time it was proposed in the paper Zhizhikina et al., 2016. This algorithm includes the following main operations: (1) wavelet decomposition of data on informative scale levels (determined during algorithm construction) is performed, and (2) spikes are detected on the basis of threshold functions (different thresholds for each scale level and for positive and negative values of the wavelet coefficients are used).

The algorithm efficiency is determined by the wavelet transform sensitivity to sharp changes of function values. Amplitudes of wavelet coefficients significantly increase in the areas containing local features in the form of sharp peaks (Daubechies, 1992). Figure 5b and e show the results of the algorithm.

The geomagnetic variations and noise are dependent on the location of the observatory. Therefore, preliminary tuning of the algorithm parameters is required for the selected observatory. Currently, the algorithm is adapted for the midlatitude Paratunka observatory and for the equatorial Hyderabad observatory. The values of the parameters were defined for the criterion of the absence of false detections, using selected data. The effectiveness of the algorithm was estimated for quiet and disturbed magnetic fields. The spikes detected by the experienced magnetologist were considered as reference. Table 2 shows the results of the estimation of the algorithm effectiveness for the Hyderabad observatory.

The results show high reliability of the wavelet-based algorithm when detecting the main part of the spikes: about $99 \%$ of spikes with high amplitude (>3 nT) are isolated. 
Table 2. The results of the estimation of the algorithm effectiveness for the Hyderabad observatory.

\begin{tabular}{|c|c|c|c|}
\hline \multirow[b]{2}{*}{ Magnetic field conditions } & \multicolumn{3}{|c|}{ Detected spikes, $\%$} \\
\hline & $\begin{array}{r}\text { Detected by } \\
\text { magnetologist }\end{array}$ & $\begin{array}{r}\text { Number of spikes: } \\
\text { Wavelet-based } \\
\text { algorithm }\end{array}$ & $\begin{array}{r}\text { Simple } \\
\text { algorithm }\end{array}$ \\
\hline Quiet (local $K<3$ ) & 1591 & 83.91 & 73.35 \\
\hline Disturbed (local $K \geq 3$ ) & 1495 & 85.35 & 73.85 \\
\hline
\end{tabular}
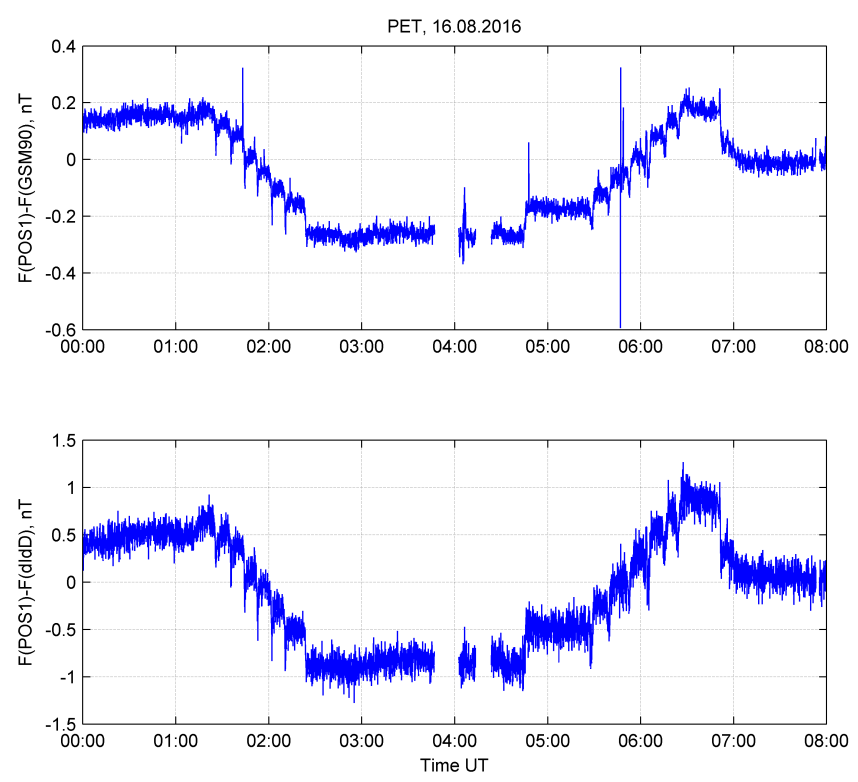

Figure 6. Example of magnetic field jumps at the Paratunka observatory during removal and lowering of casing steel pipe into a well of $80 \mathrm{~m}$ depth. Curves on the plots are differences of field total intensity $F$ measured by scalar magnetometers POS-1, GSM-90 and $\mathrm{dIdD}$ at various distances from the well. Each step corresponds to the operation with one pipe section.

However, spikes with small amplitude $(<0.5 \mathrm{nT})$, which are $25 \%$ from all spikes, cannot be reliably detected by magnetologists (experts) and cannot be used for the estimation of the algorithm effectiveness. This also restricts the possibilities for the optimization of the algorithm for detecting small spikes.

\subsubsection{Jumps}

In a certain sense, the jumps in the results of measurements can be considered to be spikes as described above, but with a continuous interval of the record between the leading and the back edges or in the absence of a back edge. Because of the sharp edges and sufficient amplitude of a jump, it is not difficult to identify one. However, unlike spikes, in practice such jumps are quite rare in raw magnetic data (for example, Fig. 2). Jumps of magnetic record level with slow changed edges or with noisy edges are predominantly ob- served. The reasons for such jumps are the technical operation with equipment, the changes of the magnetic field distribution in the pavilion or near it, the changes of the instrument parameters, etc.

An example of noise in the form of jumps caused by changes in the magnetic environment near the pavilions at the Paratunka observatory is shown in Fig. 6. The effect is visible in the field total intensity $F$, which occurred during removal and reinstallation of casing pipes in a well of $80 \mathrm{~m}$ in depth, located approximately at a distance of $100 \mathrm{~m}$ to the south from the magnetic pavilions. These operations were carried out within $6 \mathrm{~h}$, and heavy machinery was used (truck crane, tractor). In general the effect does not exceed $1 \mathrm{nT}$, but it stands out well from the difference between the records of the two scalar magnetometers, located at different distances from the well (dIdD is the closest, POS-1 is the most remote, the distance between them is $30 \mathrm{~m}$ ). Each removal of a pipe section from the well causes a jump of the magnetic field gradient between POS- 1 and dIdD by about $0.1 \mathrm{nT}$. Lowering back into the well looks like a recovery process in $\mathrm{d} F$. A much smaller effect is observed in the difference between records from the POS-1 and GSM-90 magnetometers, located at about the same distances from the well. This example represents a situation which is quite widespread at magnetic observatories and shows the following important points:

a. Identification of signals of a small amplitude by mathematical methods of pattern recognition, even if they are rather different from the field natural variations, can be practically implemented in very rare cases (only for typical noise and in the case of large samples of a priori data).

b. Such signals can be identified reliably only in difference data obtained by separated magnetometers.

c. Practically the only way to detect such signals is through an experienced, trained magnetologist, whose work is largely based on the additional information about measurement conditions.

In relation to the example given in Fig. 6, we can assert that only a magnetologist (expert) can recognize field variations as noise, by analysing the differences between measurement 

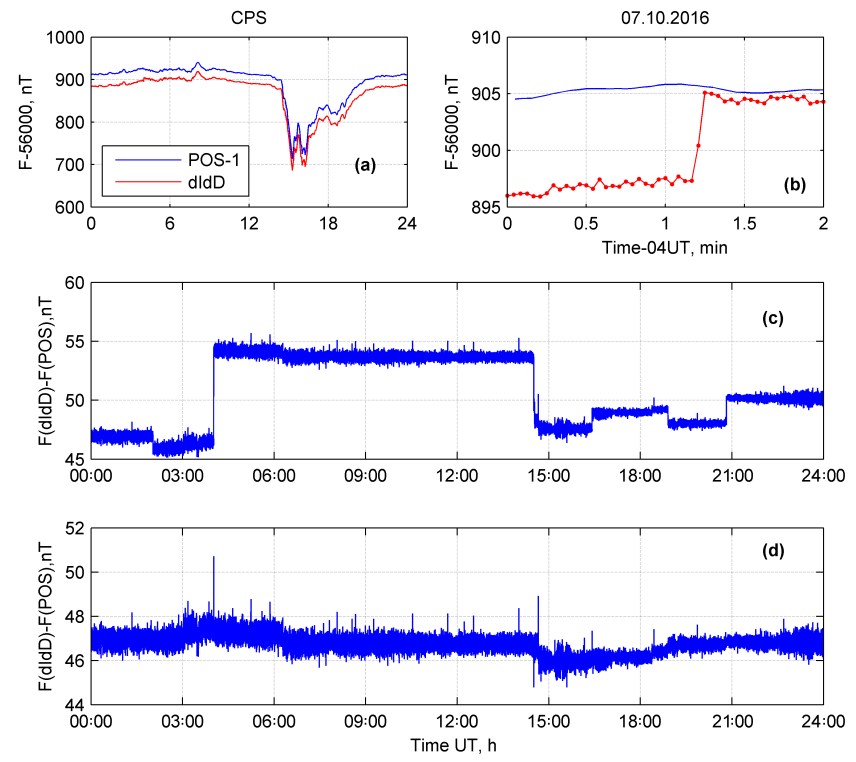

Figure 7. Example of noise in the form of jumps in the data from the dIdD GSM-19FD magnetometer at the Cape Schmidt observatory. (a) Daily records of $F$ of dIdD and scalar Overhauser magnetometer POS-1; (b) detailed fragment from (a) with a jump; (c) differences $\mathrm{d} F=F(\mathrm{dIdD})-F(\mathrm{POS})$ in which natural geomagnetic variations are removed; (d) the same as (c), but jumps in $F$ (dIdD) are removed.

results obtained by the spatially separated devices, noting artificiality of jumps in the variations and knowing that works on the well were carried out at that time. Such anthropogenic disturbances are usually not corrected, and in most cases the record including the noise is removed.

Figure 7 shows an example of jumps in the daily record of the field total intensity $F$, obtained by dIdD GSM-19FD and POS-1 magnetometers at the Cape Schmidt observatory. It can be seen in Fig. 7c that the measurement difference between these two Overhauser magnetometers contains jumps with an amplitude of several nT. However, noise arises only in the dIdD record (Fig. 7b) and in most cases have sharp edges. The magnetometers are installed in different pavilions (dIdD is in a variational, POS-1 is in absolute), at a distance of about $30 \mathrm{~m}$. The situation shown in Fig. 7c is rather characteristic of the observatory and occasionally it reoccurs. The source of the noise is not defined, but perhaps it is associated with interference of supply lines or communication cables in the variation pavilion or with the currents in moisture saturated soil near the dIdD.

In this example, our interest is not in the cause or mechanisms of noise, but in the possibility of its identification and correction. Since the amplitude is fairly significant, edges are sharp, and measurements are not burdened by this noise. The posed problem can probably be effectively solved by software tools. However, in this case, processing is performed by a magnetologist, who estimates the size and the location
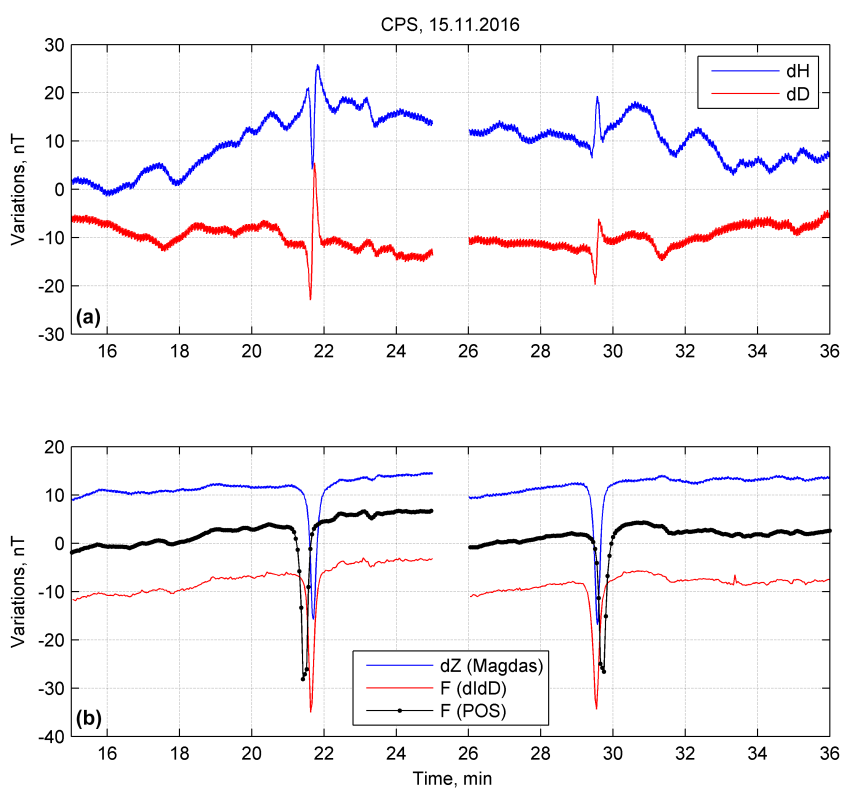

Figure 8. Noise caused by a vehicle driving near the magnetic pavilions at the Cape Schmidt observatory (in one direction and back). (a) $\mathrm{d} H$ (upper curve) and $\mathrm{d} D$ (lower curve) variations, recorded by MAGDAS magnetometer. (b) $\mathrm{d} Z$ (MAGDAS, upper curve), $\mathrm{d} F$ (POS-1, middle curve with dots) and $\mathrm{d} F$ (dIdD, lower curve). MAGDAS and dIdD magnetometers are located at a distance of about $30 \mathrm{~m}$ from the POS-1.

of the jump using an $F$ (dIdI)- $F$ (POS) plot, followed by programme correction of $F(\mathrm{dIdD})$ and necessary removal of unreliable data at the time of the jump if it has a significant duration. Figure $7 \mathrm{~d}$ shows the results of the procedure described above.

It should be noted that the jumps, after which the record level is changed and retained for a long time (several days or longer, for example, after magnetometer reinstallation), appear in the baseline values of the variometers and are eliminated during calculation of the total field vector using the standard measurement technology at magnetic observatories (see also some remarks in Sect. 4).

\subsubsection{Bay-like noise}

Bay-like noise is a common type of noise at magnetic observatories. It is often the result of changes in the magnetic field near the magnetometer due to moving objects with magnetic effect, for example a car or a person with instruments. In the case of such noise, shapes of signals in the field components are defined and related. Nevertheless, this noise, if its amplitude does not reach extreme values, is hardly distinguishable from natural variations. The possible methods of identification are comparison with data obtained by other magnetometers (gradiometer principle) and analysis of the information about events at an observatory (logging of such events is the direct responsibility of the observatory and its staff). 


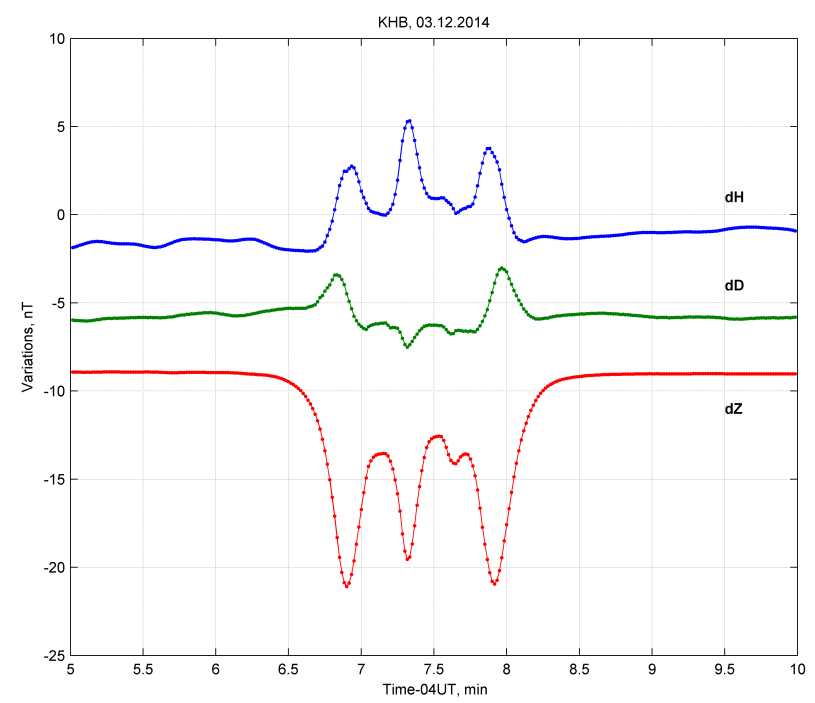

Figure 9. Noise caused by a snow plough clearing the path to the pavilions at the Khabarovsk observatory. Variations $\mathrm{d} H, \mathrm{~d} D$ and $\mathrm{d} Z$ were recorded by quartz magnetometer CAIS. Oscillations on the records are the result of the tractor work at a distance of about $30 \mathrm{~m}$ from the magnetometer.

Figure 8 shows bay-like noise occurring during off-road driving near the magnetic pavilions of the Cape Schmidt observatory and during the vehicle's return. Variations of $\mathrm{d} H$, $\mathrm{d} D$ and $\mathrm{d} Z$ components are obtained with a MAGDAS magnetometer. Variations of field total intensity $F$ are obtained using dIdD and POS-1 magnetometers (Overhauser sensors). MAGDAS and dIdD are located in a variation pavilion, POS1 is installed in an absolute pavilion. The distance between the pavilions is about $30 \mathrm{~m}$. Noise duration is about $30-40 \mathrm{~s}$, the amplitude is up to $20-30 \mathrm{nT}$, and the time shift between signals recorded by magnetometers in two remote pavilions is clearly defined. We may also note the dependence mentioned above between the form of a signal in different field components. The identification of a signal as noise was made by a magnetologist using the shape of a signal, time shift and by comparing it with similar signals that have been observed earlier (in this case there was no information about the source of the noise at the observatory). After localization, the noise was removed by a magnetologist during raw data processing.

One more example is shown in Fig. 9. A snow plough cleared the path to the magnetic pavilions at the Khabarovsk observatory. Field variations were recorded by the quartz magnetometer CAIS. Noise duration is about 1 min, amplitude is maximum in the vertical component (up to $10 \mathrm{nT}$ ). It would be difficult for the staff of the observatory to identify the signal in Fig. 9 as noise without information on the works conducted near the pavilions, because according the signal parameters, it is rather close to natural geomagnetic variations (an exception is possible only for $Z$ component, since its natural variations on this day did not exceed several
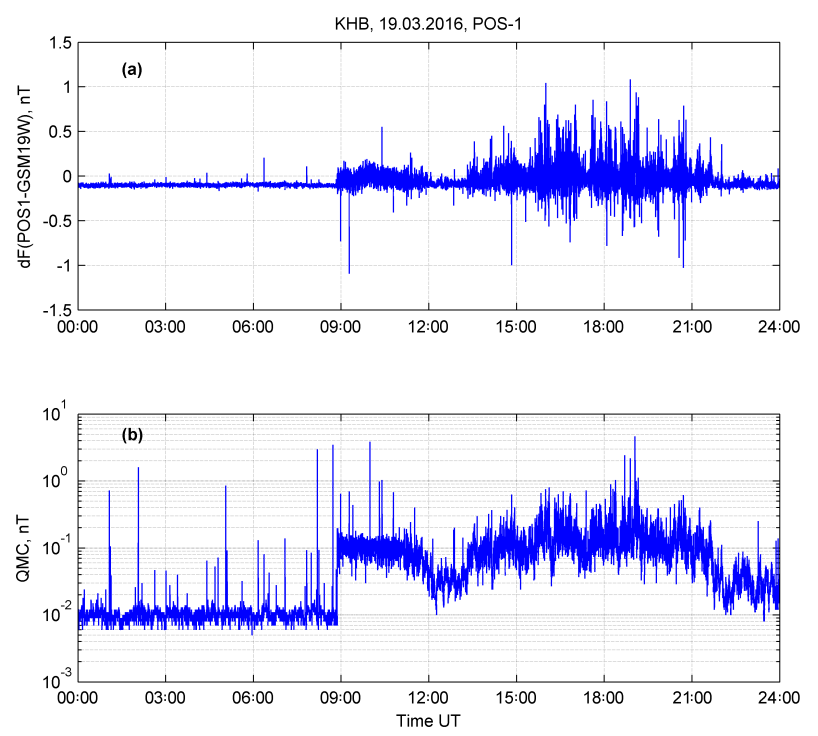

Figure 10. Noise-like interference caused by the faulty power supply at the Khabarovsk observatory, manifested in the records of Overhauser magnetometer POS-1. (a) Daily record of $F$ (POS-1) after elimination of natural geomagnetic variations by GSM-19W data; (b) behaviour of QMC (quality measurement criterion) instrumental parameter of POS-1.

of nT). Just like in the previous case, the noise was removed by a magnetologist during raw data processing.

\subsubsection{Random-like noise}

Man-made disturbances, which are expressed as additional random noise at the background of original useful signal will be understood as random noise. This notion generalizes a very big class of noise which is often difficult to classify. In many cases this noise is not localized in frequency and/or time domain. They may have a hardware origin or may be connected with real field noisiness from external sources at the observatory. Almost always, the problems of this noise is solved either technically (fine adjustment of magnetometers, improvement of grounding, power supply, etc.) or organizationally (moving of a measurement point, replacement of a magnetometer by another one which is less sensitive to noise, etc.). It is hardly probable to find effective methodological and software approaches.

Identification of hardware noise in data is possible if we compare the results of measurements by different magnetometers or if we change the operating modes of a device if it is the only one at the observatory and if it is possible by the specification of a devices. The increased noise of the magnetic field at the observatory (as a result of total impact of many factors) can be identified, for example, if we make measurements by the same magnetometer at the observatory and in the place with obviously low noise. In general, assessment of the background noise is a labour-consuming task 

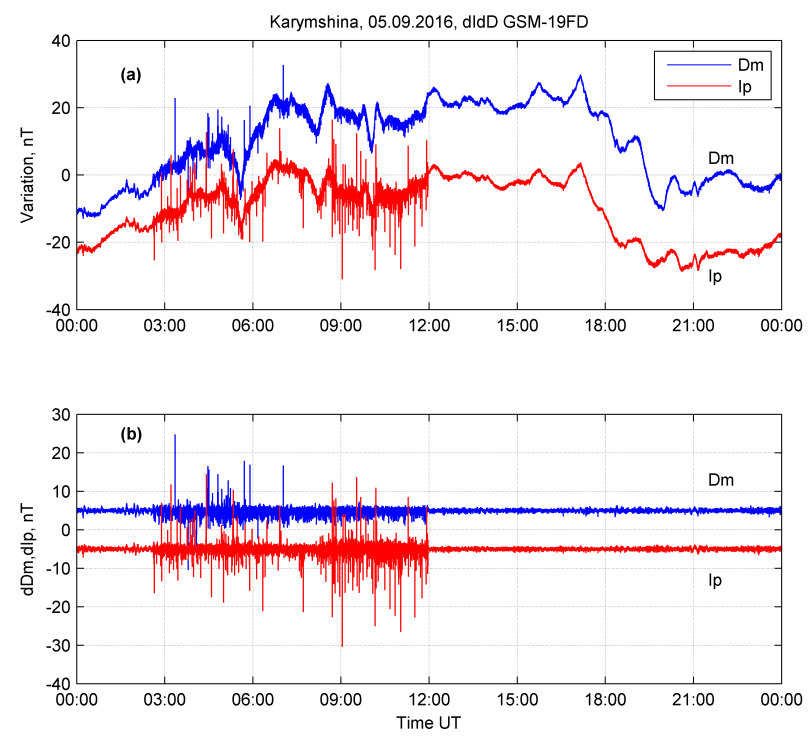

Figure 11. The noise in dIdD data which arose due to the failure of a power supply system at Karymshina station $(15 \mathrm{~km}$ from the Paratunka observatory). (a) The total field intensity $F$ obtained in the case of additional fields with polarity west-east (Dm) and updown (Ip) in the coil system (see dIdD Instruction Manual, 2010); (b) the same as in (a), but slow variations are excluded (for clarity).

including a research aspect and very often it does not have effective practical results. The problem becomes more complicated due to the fact that in many cases it is almost impossible to distinguish the noise background from a natural signal which is a subject of scientific research, for example, seismomagnetic effects. Figures 10 and 11, as an example, show the random noise which arises in the results of magnetic measurements in the case of problems with power supply.

Figure 10 shows the results of daily measurements of the field total intensity $F$, made at the Khabarovsk observatory using scalar Overhauser magnetometer POS-1. There was a general power outage at the observatory and in the nearby settlements at about 08:50 UT. The measurements were continued using an autonomous power supply system at the observatory. At about 12:00 UT the external power supply was restored. On the record obtained by POS-1, noise with an amplitude up to $1 \mathrm{nT}$ was recorded. It lasted for more than a day. For a descriptive graphical representation, natural geomagnetic variations were excluded using the data from another scalar magnetometer (GSM-19W). The noise caused by power failure did not manifest in these measurements (Fig. 10a). Figure 10b shows the behaviour of the QMC parameter, which estimates the quality of the precession signal of the POS-1 Overhauser sensor in nT units and was described in Sect. 3.2.1. In this example, the fact that the noise remained in the measurements of POS- 1 after the restoration of external power is of interest; i.e. the presence of the noise could not be ascertained according to staff information on the situation at the observatory, it could be determined only by the direct visual analysis of these measurements. And the second fact is that in this case an effective way to recognize the noise is to estimate the behaviour of the QMC parameter.

The second example (Fig. 11) shows an appearance of noise in the data from the dIdD GSM-19FD magnetometer, recorded at Karymshina station of IKIR FEB RAS. Karymshina station is located approximately $15 \mathrm{~km}$ from the Paratunka observatory, a place with a minimum of possible industrial sources of noise, including the absence of an external power supply by power lines. At about 02:45 UT a failure of a diesel generator occurred and an emergency scheme of power supply has been activated with an external battery package as a source of voltage and disconnection from all powerful devices, the standard power supply was restored at about 11:55 UT. It is shown in Fig. 11a that spikes with amplitude up to 10-20 nT and random noise up to $5 \mathrm{nT}$ appeared during the operation of the emergency power system in two measurement channels of dIdD (with additional fields in coil systems D and I; see, for example dIdD Instruction Manual, 2010). For illustration purposes, Fig. 11b shows the record after eliminating the low-frequency variations. Since the amplitude of the noise is significant, it can be easily identified during the analysis.

In both examples given above, the only way to exclude noise is to remove all data fragments which contain noise. It is impossible to restore the original useful signal with an acceptable quality.

\subsection{Critical and weak noise}

This criterion is important due to the fact that it determines the extent of noise influence in magnetic data on the results of research that is carried out using these data. When a researcher uses the final data from the observatories, in most cases they have no information on what conditions the measurements were performed under, what procedures were applied during preliminary processing, etc. (except for the cases when these procedures are prescribed by standards). Thus, the responsibility for the quality of the data provided to the scientific community is very high and it lies entirely on the observatory.

Let us consider a classic case. An observatory of the INTERMAGNET network obtains primary $1 \mathrm{~s}$ data of $\mathrm{d} H \mathrm{~s}$, $\mathrm{d} D \mathrm{~s}, \mathrm{~d} Z \mathrm{~s}, F \mathrm{~s}$ variations by direct measurements and then, using baseline values, calculates total $H \mathrm{~s}, D \mathrm{~s}, Z \mathrm{~s}, F \mathrm{~s}$, reduced to the main pillar and minute values $H \mathrm{~m}, D \mathrm{~m}, Z \mathrm{~m}$, $F \mathrm{~m}$ using the procedure defined by INTERMAGNET standards (INTERMAGNET Tech. Ref. Manual, 2012). Filtering using a Gaussian filter is quite an effective method for suppressing random noise. Also, Gaussian filtering works acceptably with spikes of small amplitude, but it is not effective for jumps and bay-like noise. Thus, the residual effects from strong noise are included into the final minute data. At the same time they are smoothed; i.e. they are almost indistinguishable against the background of natural variations, but 


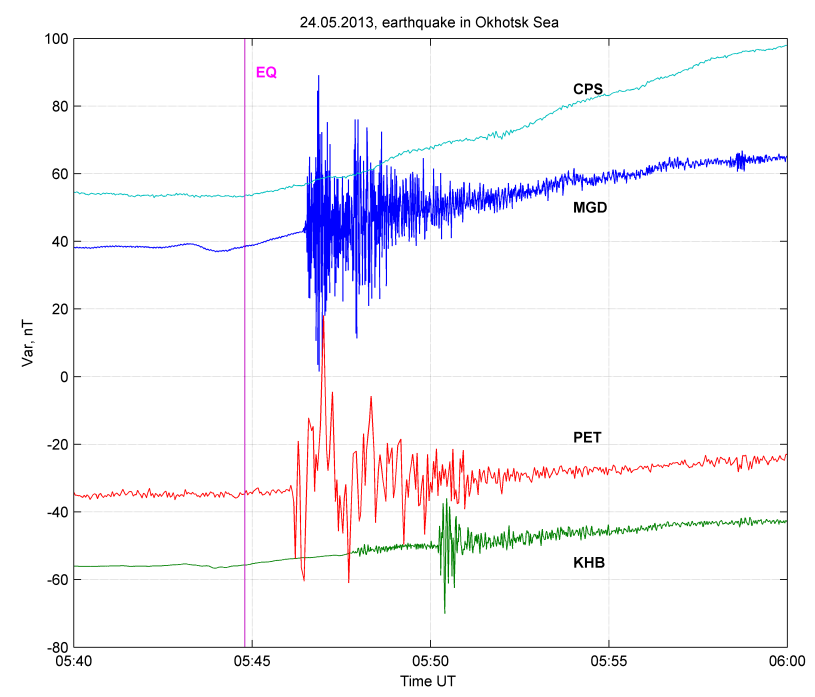

Figure 12. "Fictitious" variations of the magnetic field recorded by magnetometers with suspension system of measuring sensors: dIdD with scalar sensor in the coil system, FGE with fluxgate sensors and magnetometer Quartz-6 with Bobrov's quartz sensors. Records obtained at CPS, MGS, PET and KHB observatories during a strong earthquake in the Okhotsk Sea on 24 May 2013 are shown. Vertical line EQ shows the earthquake time.

they influence the results of further calculations performed with these data. That makes it necessary to identify such critical noise and to remove it during the primary data processing. It entails secondary problems, such as gaps in original data, which are used to calculate published minute values. In spite of the INTERMAGNET recommendation that mean values should be calculated in accordance with the $90 \%$ availability rule: this rule is difficult to define and sometimes not applicable at all.

\subsection{Noise with known and unknown sources}

If it is known that a signal, which is suspected as noise, is the result of some sources which are not associated with natural variations of magnetic field, then there is a rather a powerful argument for removing the signal. At the same time, however, there are some fine points:

a. noise removal leads to the gaps in published data, therefore scientist in many cases before application of methods of analysis is forced to fill these gaps with dummy data, i.e. the data, calculated from available data set using some interpolation method. Errors of calculation results arising due to the filling may be comparable or even greater than the errors that occur due to the noise which was not deleted;

b. problems which are solved using the final data should be defined. Some signals can be as noise due to the criteria of its origin. However, at the same time these signals can be the subject of other scientific research. For example, in practice of observatory measurements, a fictitious "seismomagnetic effect" is well known, when we observe oscillations in the data from magnetometers with a suspended system for compensation of sensor inclinations or in the records of induction magnetometers. These oscillations arise when a seismic wave from a near or strong earthquake is passing the place where the magnetometer is installed. As an example, Fig. 12 shows the records of magnetic field variations at IKIR FEB RAS observatories. On these fragments the effect of a strong earthquake with the magnitude of 8.3 which occurred on 24 May 2013 in the Sea of Okhotsk at the depth of about $600 \mathrm{~km}$ is illustrated. The following magnetometers were applied: dIdD GSM-19FD (Dm and Im modules with additional fields of coil system) at the CPS and PET, fluxgate FGE ( $H$ channel) at MGD, digital magnetometer with Bobrov's quartz sensors ( $H$ channel) at KHB. It is clear that the earthquake is manifested well at long distances, the magnetometer data based on different measurement principles. For the tasks of studying the variability of the magnetic field, the signals shown in Fig. 12 are noise and they should be removed. However, if, for example, seismomagnetic effects are investigated, then the recorded "fictitious" signals in Fig. 12 would be a good benchmark to estimate the passage of a seismic wave in the area where magnetometers are installed. Therefore, the researcher of seismomagnetic effects should understand well, what type of magnetometer is used to obtain magnetic data.

If a signal is suspected as a noise, but its structure does not allow us to recognize it as a noise, and there is no additional information about a possible source, then it is very complicated for a magnetologist to make a decision. In most cases, these signals are not removed, which creates risks of reducing the quality of observatory data.

\section{Possible methods for noise removal from raw magnetic data}

It is clear that identification of noise is the solution only of a part of the problem. The second part is to choose an effective method of further work with this noise. Unfortunately, the choice of possibilities is very small. Mainly a fragment is simply removed from a record that results in a gap, which can be filled by the most suitable "dummy" data, or if the structure of the noise is recognized, it is removed and the original useful signal remains.

Earlier, in the description of spikes (see Figs. 2, 4 and 5), possible techniques of dealing with these noise, using automatic removal of a record fragment after identification of noise, have already been shown. In other cases, noise can be removed manually by a magnetologist. There are different approaches to implement these procedures. If a file with 


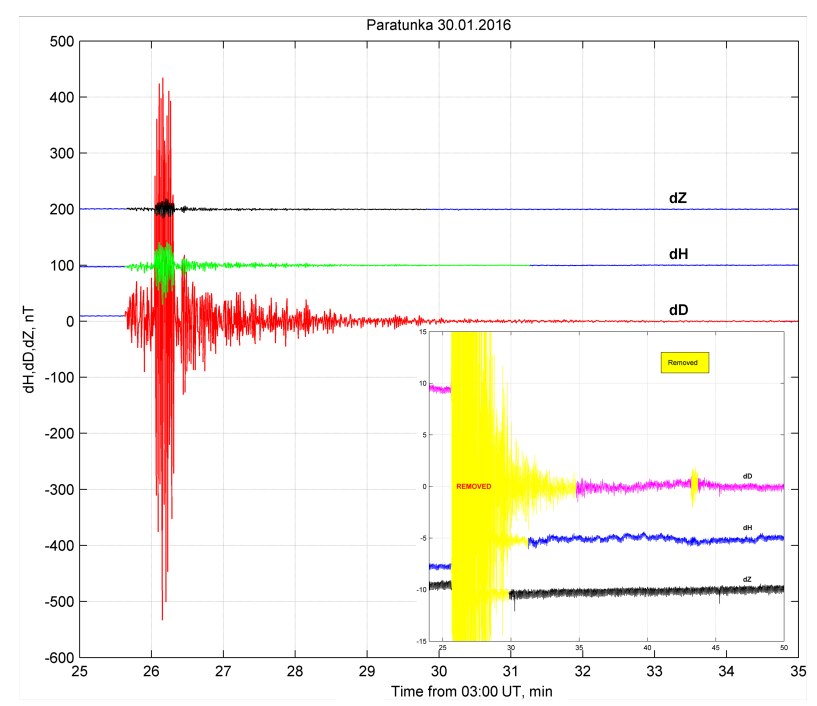

Figure 13. Example of manifestation of a nearby earthquake on 30 January 2016 (magnitude 5.7, distance is about $100 \mathrm{~km}$ ) in the record of FGE magnetometer with a suspended system at the Paratunka observatory. The inset shows the results of data clearing performed by a magnetologist using a semi-automatic procedure (shown in yellow). In component $D$ the effect of an aftershock, which occurred in $17 \mathrm{~min}$, is also removed. Jumps of average level of the records, reaching $10 \mathrm{nT}$ in $D$ (about $1.5^{\prime}$ ) are clearly visible in the inset.

raw data has text format (for example, POS-1 User manual, 2004 and dIdD Instruction Manual, 2010), it is possible to remove the unreliable records manually. In this case, during further work with the corrected file, there will be gaps not only in the measured magnetic values, but also in a timestamp, which is not always convenient if we need a uniform time grid. Another possible approach is to create an intermediate file, usually in text format, in which noisy data are manually or semi-automatically replaced by the values that indicate unreliable measurements, for example 99 999. This option is particularly useful if the initial measurements are recorded in files in binary format, e.g. MAGDAS-A Installation Manual (2005). In this case special converter software is usually used, including those which allow an operator to encode the required data as unreliable.

In the software for magnetologists developed in IKIR FEB RAS, which is based on MATLAB and Octave mathematical packages (Khomutov, 2016), the third approach is used. It is based on the following methodical principles: (a) original files are always used in processing of raw (original) magnetic data, and (b) the intermediate files are not formed; i.e. though stream processing is performed from the raw data to the required final result, all the results of intermediate computations remain only in the computer's random access memory. Information about unreliable data during a time interval is stored in special text files, with a date, start time and end time and special features, for example for the choice
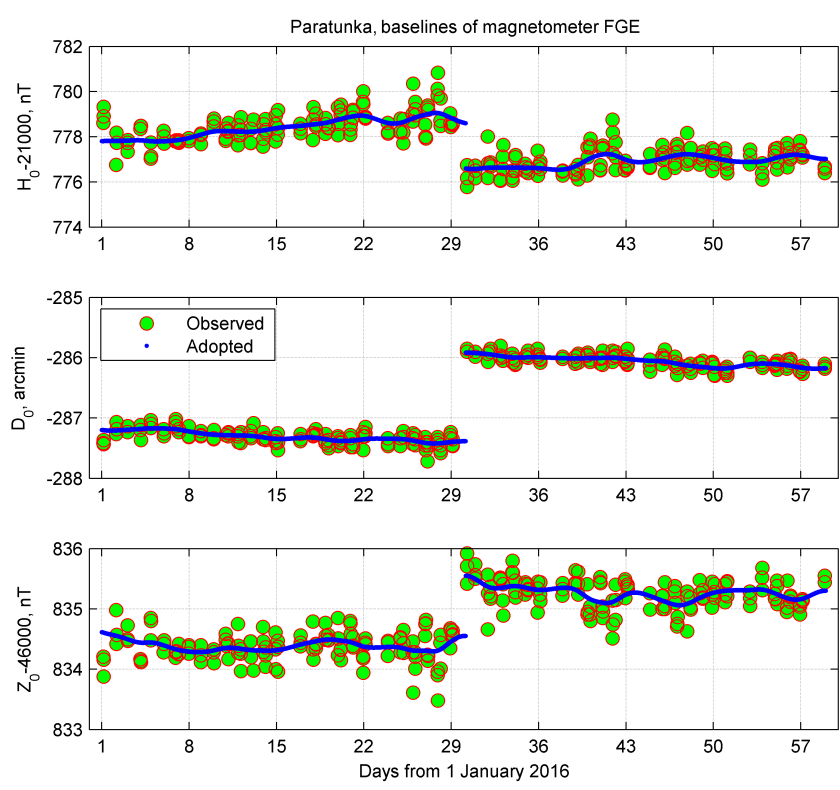

Figure 14. Baseline values of FGE variation magnetometer, Paratunka observatory, from January to February 2016. Marker $\bigcirc$ shows individual baseline values (observed); continuous curve shows the values adopted for each minute (adopted). The jumps in baselines due to the earthquake on 30 January 2016 are clearly visible.

of the magnetic components and for comments. An example of such a record for the FGE magnetometer at the Paratunka observatory is shown below.

\begin{tabular}{lrrl} 
Date & UT1 & UT2 & HDZ \\
\hline 30 Jan 2016 & 03.4272 & 03.5211 & $100 \%$ earthquake \\
30 Jan 2016 & 03.4269 & 03.5795 & $010 \%$ earthquake \\
30 Jan 2016 & 03.4274 & 03.4973 & $001 \%$ earthquake \\
30 Jan 2016 & 03.7199 & 03.7283 & $010 \%$ aftershock
\end{tabular}

A maximally simplified text format is used, which reduces the probability of errors during manual typing and speeds up the file reading. The example shows the information, used to remove the noise caused by a nearby earthquake on 30 January 2016 in the data from the FGE magnetometer. In the processing of measurement results from 30 January $2016, D$ values from $03: 25: 37(=03.4269)$ to 03:34:46(=03.5795) UT, which are noisy due to the mechanical influence of a seismic wave on the suspended sensor, will be filled with a special symbol, $\mathrm{NaN}$, and will be excluded from further processing. The boundaries of intervals with noise are defined by a magnetologist in interactive mode by record plots for the corresponding components and with required scaling that is provided by convenient interface with graphics in MATLAB and Octave. Noise in other components is removed similarly. The effect of the described earthquake and the results of applying the clearing procedure are shown in Fig. 13. 
Considerably a more difficult situation arises in the attempt to restore an original signal, i.e. removal only of noise from the measurement results. It is impossible in most cases in the actual working practice of the observatory due to the unknown structure of a useful signal and the unknown structure of noise. Analysis of a concrete situation is specific, and become a scientific research question in itself. However, in some simple cases the problem can be solved. These situations include, for example, noise, leading to jumps of record level, sometimes followed by restoration which is also in the form of a jump. It is clear that in this case the noise is a constant addition to the useful signal, and after its subtraction, the initial undisturbed signal will be restored. The main problem is the correct estimation of the value of the noise (magnitude of a jump) and the need to be sure that noise has a constant value in the analysed interval of the record.

In practice, identification of a jump and estimation of its parameters (time and magnitude) is performed by a magnetologist, usually in an interactive mode of work with magnetic record graphs. If the jump was very rapid and the time of a transitional process was comparable with the duration of the interval between samples and generally faster than possible natural variations in the magnetic field, then the jump parameters can be estimated visually with sufficient reliability. If the transitional process is long enough, then natural variations in the field can significantly affect the accuracy of the estimation. In this case, it may be useful to compare the analysed record with measurement results obtained by another magnetometer, because natural geomagnetic variation will be excluded from the difference between two records, and the jump will be manifested as two permanent levels of the difference. However, in this case, there are also a lot of limitations, for example the nature of the cause of the jump. If the jump is caused by a change in the magnetic field, the source of this change is fairly close to the place of measurements, and the compared magnetometers are located closely (usually in the same or in neighbouring pavilions), then noise effect will be manifested in the data from both devices and it will be difficult to make any reliable estimations. If the reason of the jump is of technical origin or its impact on the supporting magnetometer is small, it is possible to estimate the required parameters.

The situation described above is partly similar to that which occurs in the case of elimination of long-term changes of the level of magnetic record from variation magnetometer, using absolute observations. Figure 14 shows an example of compensation of jumps that arose in the records of the FGE magnetometer at the Paratunka observatory after the earthquake on 30 January 2016 (see Fig. 13). Regular absolute magnetic observations and dense series of obtained baseline values allow us to effectively eliminate the effect of such jumps in the series of full values of the magnetic field components; for example, the total values of the declination $D=D 0+\mathrm{d} D$ since 31 January 2016 will have been free of the jump effects in the case of an earthquake.

\section{Conclusions}

The review of noise in raw magnetic data and some methods of its identification and removal given above represent only a part of the real situation which a magnetologist deals with at an observatory when processing the measurement results. However, even with such a volume it is possible to draw the following important conclusions:

1. In most cases, the correct identification of noise can be performed only according to the estimates made by an expert, a magnetologist of the observatory, who uses raw (original) results of measurements, and all available additional information on the measurement conditions.

2. The most important source of information about noise is the comparison of measurement results obtained by different magnetometers, including those using different measuring principles, as well as careful monitoring of the environment at the observatory.

3. Automatic identification and correction of noise by computer programs are of auxiliary nature and are principally an interactive tool used to help a magnetologist with data processing.

All this indicates that the data processing should be carried out by a qualified magnetologists directly at a workplace, i.e. at the observatory, and should include the whole set of requirements (Jankowski and Sucksdorff, 1996; Nechaev, 2006; INTERMAGNET Tech. Ref. Manual, 2012). A similar opinion is presented by Linthe et al. (2012). Unfortunately, at the present time, many observatories, especially those that are newly created and located in remote areas, have problems with staff and their qualifications. In these cases it seems reasonable to create centres for collecting raw magnetic data, where a full cycle of data processing would be performed. Examples of such centres are BGS, which has a centre in Edinburgh (http://www.geomag.bgs.ac.uk/data service/space_weather/current_conditions.html) or GC RAS (http://geomag.gcras.ru/) which collects and processes the data from the Russian magnetic observatories. At the same time, some of the problems, including those connected with incorrect noise processing, can be more difficult to resolve.

Data availability. All raw magnetic data used in this study are available at the observatories of IKIR FEB RAS and CSIR-NGRI. Some data are available on request.

Competing interests. Kusumita Aurora is a member of the editorial board of the special issue of the journal.

Special issue statement. This article is part of the special issue "The Earth's magnetic field: measurements, data, and applications 
from ground observations (ANGEO/GI inter-journal SI)". It is a result of the XVIIth IAGA Workshop on Geomagnetic Observatory Instruments, Data Acquisition and Processing, Dourbes, Belgium, 4-10 September 2016.

Acknowledgements. The authors are grateful to the staff at the observatories of IKIR FEB RAS for providing qualitative magnetic measurements. Stanislav Nechaev, main magnetologist of Patrony observatory (Irkutsk, IRT), Pavel Borodin, magnetologist of Arti observatory (Arti, Ekaterinburg, ARS), Zinaida Dumbrava, Head of Khabarovsk observatory (IKIR FEB RAS, KHB) and Vladimir Sapunov, Head of Quantum Magnetic Laboratory (Ural Federal University, Ekaterinburg) are acknowledged for helpful discussions over many years, which improved our understanding of noise in magnetic observations. Phani Chandrasekhar and K. Chandashakhar Rao are acknowledged for their contributions to the observatories in Hyderabad and Choutuppal. The director of CSIR-NGRI is acknowledged for his permission to publish this work. The DST-RFBR collaboration is acknowledged for funding the joint studies (Grant of DST no. INT/RUS/RFBR/P-234, dated 28-9-2016 and Grant of RFBR no. 16-55-45007). Magnetometers POS-1 and POS-4 for the observatories of IKIR FEB RAS were purchased with grant funds of the Russian Science Foundation, project number 14-11-00194. The authors are very grateful to Anna Larionova for her help with English corrections, to the referees and the editorial advisor for the comments and corrections made in the manuscript. They greatly improved the quality of presentation.

Edited by: Alexandre Gonsette

Reviewed by: Christopher Turbitt and one anonymous referee

\section{References}

3 component fluxgate magnetometer FRG-601G: Ver1.1. Operation manual, Tierra Tecnica Ltd, p. 9, 2002.

Auster, H. U., Mandea, M., Hemshorn, A., Korte, M., and Pulz, E.: GAUSS: Geomagnetic Automated System, Proceedings of the XII IAGA Workshop on Geomagnetic Observatory Instruments, Data Acquisition and Processing, Belsk, 19-24 June 2006, Publication of the Institute of Geophysics Pol, edited by: Reda, J., Acad. Sci., 398, 51-61, 2007.

Daubechies, I.: Ten Lectures on Wavelets, SIAM, 377 pp., 1992.

Denisov, A. Y., Denisova, O. V., Sapunov, V. A., and Khomutov, S. Y.: Measurement quality estimation of proton-precession magnetometers, Earth Planets Space, 58, 707-710, 2006.

dIdD v.7.0 Insrtuction Manual: GEM Systems, Canada, 50 pp., 2010.

GSM-19 v7.0 Instruction Manual: GEM Systems, Canada, 149 pp., 2008.

Hegymegi, L., Csontos, A., and Merenyi, L.: Monitoring of long term mechanical stability of a suspended dIdD sensor applying optical observation, J. Ind. Geophys. Union, 2, 19-23, 2016.

INTERMAGNET technical reference manual: Version 4.6, edited by: St Louis, B., 92 pp., available at: http://intermagnet.org/ publications/intermag_4-6.pdf (last access: 7 February 2017), 2012.
Jankowski, J. and Sucksdorff, C.: Guide for magnetic measurements and observatory practice, Warsaw, 235 pp., 1996.

Khomutov, S. Y.: Methodological and software approaches to processing of magnetic measurements at observatories of IKIR FEB RAS, Russia, J. Ind. Geophys. Union, 2, 54-61, 2016.

Khomutov, S., Sapunov, V., Denisov, A., Savelyev, D., and Babakhanov, I.: Overhauser vector magnetometer POS4: Results of continuous measurements during 20152016 at geophysical observatory "Paratunka" of IKIR FEB RAS, Kamchatka, Russia, E3S Web Conf., 11, 1-5, https://doi.org/10.1051/e3sconf/20161100007, 2016.

Linthe, H.-J., Reda, J., Isac, A., Matzka, J., and Turbitt, C. W.: Observatory Data Quality Control - the instrument to ensure valuable research, in: XVth IAGA Workshop on Geomagnetic Observatory Instruments and Data Processing, Cadiz, Spain, 4-14 June 2012, 173-177, 2012.

Lopez-de-Lacalle, J.: tsoutliers R Package for Detection of Outliers in Time Series, available at: https://jalobe.com/doc/tsoutliers.pdf (last access: 20 January 2017), 2016.

MAGDAS-A Installation Manual, SERC, Kyushu Univ., edited by: Maeda, G. N., 35 pp., 2005.

Maule, C. F., Thejll, P., Neska, A., Matzka, J., Pedersen, L., and Nilsson, A.: Analyzing and correcting for contaminating magnetic fields at the Brorfelde geomagnetic observatory due to high voltage DC power lines, Earth Planets Space, 61, 1233-1241, 2009.

Nechaev, S. A.: Manual for stationary geomagnetic observations, Publ. Institute of Geography Siberian Branch of RAS, Irkutsk, 140 pp., 2006 (in Russian).

Nelapatla, P. C., Kumar Potharaju, S. V., Arora, K., Kasuba, C. S. R., Rakhlin, L., Tymoshyn, S., Merenyi, L., Chilukuri, A., Bulusu, J., and Khomutov, S.: One second vector and scalar magnetic measurements at low latitude observatory, CPL, Geosci. Instrum. Method. Data Syst. Discuss., https://doi.org/10.5194/gi2017-16, in review, 2017.

Neska, A., Reda, J., Neska, M., and Sumaruk, Y.: On the influence of DC railway noise on variation data from Belsk and Lviv geomagnetic observatories, Acta Geophys., 61, 385-403, https://doi.org/10.2478/s11600-012-0058-0, 2013.

POS-1: Processor Overhauser Sensor - Magnetometer, User Manual, Ural State Technical University, 21 pp., 2004.

Santarelli, L., Palangio, P., and De Lauretis, M.: Electromagnetic background noise at L'Aquila Geomagnetic Observatory, Ann. Geophys.-Italy, 57, G0211, https://doi.org/10.4401/ag6299, 2014.

Sapunov, V. A., Denisov, A. Y., Saveliev, D. V., Soloviev, A. A., Khomutov, S. Y., Borodin, P. B., Narkhov, E. D., Sergeev, A. V., and Shirokov, A. N.: New vector/scalar Overhauser DNP magnetometers POS-4 for magnetic observatories and directional oil drilling support Magnetic Resonance in Solids, Electron. J., 18, 16209, 2016.

Turbitt, C., St-Louis, B., Rasson, J., Matzka, J., Stewart, D., Lalanne, X., Schwarz, G., and Shanahan, T. INTERMAGNET Definitive One-second Data Standard, INTERMAGNET Technical Note, ver.1.0, TN6, 7 pp., 2014.

Zhizhikina, E. A., Mandrikova, O. V., and Khomutov, S. Y.: Algorithm for detection of artificial disturbances in geomagnetic data, Bulletin of Kamchatka State Technical University, 35, 21 26, 2016 (in Russian). 